\title{
Effects of Somatosensory Stimulation Therapy in Improving Upper Limb Function of Patients with Hemiplegic Stroke
}

\author{
Sang-Mi Jung, OT, PhD*1 \\ ${ }^{* 1}$ Dept. of Occupational Therapy, Sangji Youngseo University, Republic of Korea
}

\begin{abstract}
Purpose The aim of this study was that tailored intervention for hemiplegic patients which include not only sensory stimulation but also active movement and manipulation would benefit on upper limb functions. Methods An experimental group (EG) of 10 subjects and a control group (CG) of 10 subjects received a somatosensory stimulation therapy (SST) and a traditional occupation therapy (Transferring cups, folding towels, swiping a desk, a ROM arc and a peg board) for 30 minutes a day, 3 times a week for 5 weeks, respectively. And, the upper limb functions were tested before and after the intervention. Results There were significant differences in Shoulder/Elbow/Forearm (SEF), Hand and Coordination in pre-post test in the EG. And, there were significant differences in SEF and Hand in pre-post test in the CG. Besides, there were significant differences in SEF, Hand and Coordination in post-pre test in between the EG and CG. Conclusion Ongoing repetition of sensory input in combination with active movement brought improved upper limb functions.
\end{abstract}

Key words antigravity muscles, proprioception, somatosensory stimulation, stroke, upper limb function

Corresponding author Sang-Mi Jung (otjsm@hanmail.net)
Received date
08 Jan 2020
Revised date
10 Feb 2020
Accept date
13 Feb 2020

\section{Introduction}

Cerebral lesion induces a variety of deficits, and somatosensory deficit is the significant consequence of it. ${ }^{1)}$ And, upper limb paresis affects negatively on regaining activities of daily living dependently. As a matter of fact, at best, $12-34 \%$ of the patients with stroke attain full functional recovery in the affected upper limb in 6 months after stroke. ${ }^{2)}$ Somatosensation decreased in $37 \%$ of patients with the right hemispheric lesion and $25 \%$ of patients with the left hemispheric lesion. And, deficit of tactile reception and manipulating an object, diminishing motor control of the affected extremity, deficits in adjusting the level of force of the hand during grasping, and reduced balance control in an upright position and during ambulation are obvious. In the upper limb case, a learned nonuse mechanism is one of the most significant reasons making the patients with stroke lose their upper limb function and hard to get functional

http:dx.doi.org/10.17817/2020.02.10.111531 recovery. ${ }^{1)}$ Notwithstanding current advances in rehabilitation, an extensive fraction of stroke patients keeps experiencing persistent upper-limb dysfunctions. Only 1 out of 5 patients would get recovered fully in arm function, yet a half of them would not recover any function in the affected arm. The upper limb function counts mainly on the recovery of sensorimotor in the affected hand. ${ }^{3)}$ Pain, anxiety and diminished sensorimotor function frequently remain after stroke and they have hard time on higher healthcare costs, impaired dependence and health. ${ }^{4)}$ Because neural process consists of planning, execution and control of motor behaviors, selecting proper sensory modalities from the body and external environment is important. In order to normal and accurate motor output, correct processing and sensory input transmit to the brain are significant before and ongoing movement. Therefore, sensory processing and motor output have a tight connection. 5) The previous study proved that somatosensory areas in the brain has innumerous capacity of neuroplasticity so that rehabilitative training focused on restoring somatosensory deficit with con- 
sequent disabilities can bring up substantial functional improvement. ${ }^{1)}$ Moreover, the previous study concretely showed that sensory stimulation led somatosensory deficit improved after brain damage. Although sensory stimulation has effect on functional outcome, it has been received less attention.) ${ }^{6}$ Therefore, sensory stimulation should be focused in the intervention in order to bring an optimal movement pattern in patients with stroke. The diverse stimuli coming through the distal part of the body would be conveyed to the brain and modified and activated the primary somatosensory areas with previous memory in the associated areas would make the best and optimal movements. So, in this study, tailored interventions for hemiplegic patients which include not only sensory stimulation but also active movement and manipulation would benefit on upper limb functions.

\section{Materials and Methods}

This study was conducted from March $4^{\text {th }}$ to April $5^{\text {th }}$, 2019. All subjects were from B hospital in Sungnam-Si, Republic of Korea. The purpose and methods were explained to the subjects before initiating this study. And, the subjects submitted the informed consent in advance. A total of 20 subjects were participated in this study. The inclusion criteria were as follows: 1) diagnosed with stroke from neurologist within 6 months, 2) understanding the instructions from the researcher, 3) having sensation in the affected side hand, 4) being able to maintain sitting or standing up for 30 minutes, and 5) no orthopedic problems.

The subjects were allocated into two different groups by a simple random sampling. An experimental group (EG) of 10 subjects and a control group (CG) of 10 subjects received a somatosensory stimulation therapy (SST) and a traditional occupation therapy (Transferring cups, folding towels, swiping a desk, a ROM arc and a peg board) for 30 minutes a day, 3 times a week for 5 weeks, respectively. The upper limb functions were tested before and after the intervention and more than 5 year clinical practitioners performed the intervention and assessment who were trained with a protocol of the intervention in advance.

\section{Somatosensory stimulation therapy}

The main idea of Somatosensory stimulation therapy (SST) is to input efficient somatosensation to the affected upper limb by making the subjects perform movements through active contact stimulation on the affected hand after giving focused sensation to it. ${ }^{1)}$

\section{The specific sequence of the intervention is as follows.}

1) A starting position: The subject put the both hands on the table with the feet flat on the ground and the both knees placed right above the feet and the back straightened up.

2) Activation of intrinsic muscles in the affected hand: Through adjusting the length of the muscles in between $2^{\text {nd }}$ and $5^{\text {th }}$, tactile sensation and proprioception were provided to the intrinsic muscles in order to strengthen the individual intrinsic muscles and improve perception on sensory input on it. ${ }^{7)}$

3) Improvement of perception through scapular movements: It is to activate the antigravity muscles and facilitate proprioception by performing movements with the therapist in order to improve scapular stability. The upper limb movement should be straight forward but rotational movement should be made with each joint in the upper limb in order to reach the arm all the way out in an effective way. ${ }^{8)}$

4) Controlling the affected arm in the air: It is to control the affected arm in the air when reaching it all the way out in order to reach or grasp a target by using controlled movement from reciprocal innervation of biceps and triceps brachii with an improved controlling skill of antigravity muscles."

5) Manipulating an object in the affected hand: It is to increase perception capacity through manipulating a variety of tactile materials with the affected hand in order to rapid perception of the tactile sensation and proprioception of each joints in the hand. ${ }^{10)}$

\section{Fugl-Meyer Assessment for the Upper limb} Fugl-Meyer Assessment for the Upper limb (FMA) is developed in order to evaluate functional recovery of 
the patients with stroke and it has .96 of reliability which is high. ${ }^{11)}$ In this study, shoulder/elbow/forearm (SEF), wrist, hand, coordination and speed in the FMA were assessed.

This study was analyzed using SPSS Windows 21.0 version. The Kolmogorov-smirnov test, Chi-square test and Mann whitney U test were used for normality distribution, general characteristics and homogeneity of the subjects, respectively. The difference of pre and post-test in the EG and CG was analyzed with Wilcoxon signed ranks test. A $\alpha=.05$ level of significance was used for the statistical test.

\section{RESULTS}

A total of ten subjects ( 6 Males and 4 Females, the mean age of $46.0 \pm 4.73$ years old, 7 hemorrhages and 3 infarctions, 4 left side lesions and 6 right side lesions) in the EG participated in this study. And, a total of 10 subjects $(7$ Males and 3 Females, the mean age of $43.4 \pm 6.04$ years old, 6 hemorrhages and 4 infarctions, 5 left side lesions and 5 right side lesions) in the CG participated in this study. There was not a significant difference in homogeneity of the subjects in between the groups.

The general characteristics are shown in Table 1.

There were significant differences in SEF, Hand and Coordination in pre-post test in the EG. And, there were significant differences in SEF and Hand in pre-post test in the CG. Besides, there were significant differences in SEF, Hand and Coordination in post-pre test in between the EG and CG. In general, the EG has improved in all categories of FMA comparing to the results of the CG. The results of the Fugl-Meyer Assessment for the upper limb in pre and post-test are shown in Table 2.

\section{DISCUSSION}

The first 4 weeks after a stroke play a very important role in terms of regaining hand dexterity like motor skills such as grasping, reaching, moving, gripping and releasing objects. What that means is that there exists a certain very important time line in order to

Table 1. General characteristics of the subjects.

$(\mathrm{N}=20)$

\begin{tabular}{|c|c|c|c|c|c|}
\hline & & $E G(n=10)$ & $C G(n=10)$ & $x^{2}$ & $\mathrm{P}$ \\
\hline \multirow{2}{*}{ Gender } & Male & $6(60 \%)$ & $7(70 \%)$ & \multirow{2}{*}{.64} & \multirow{2}{*}{1.00} \\
\hline & Female & $4(40 \%)$ & $3(30 \%)$ & & \\
\hline \multicolumn{2}{|c|}{ Age (years) } & $46.0 \pm 4.73$ & $43.4 \pm 6.04$ & .53 & .99 \\
\hline \multirow{2}{*}{ Stroke } & Infarction & $3(30 \%)$ & $4(40 \%)$ & \multirow{2}{*}{.64} & \multirow{2}{*}{1.00} \\
\hline & Hemorrhage & $7(70 \%)$ & $6(60 \%)$ & & \\
\hline \multirow{2}{*}{ Lesion side } & Left & $6(60 \%)$ & $5(50 \%)$ & \multirow{2}{*}{.65} & \multirow{2}{*}{1.00} \\
\hline & Right & $4(40 \%)$ & $5(50 \%)$ & & \\
\hline
\end{tabular}

n (\%) / M $\pm S D ; E G$, Experimental Group; CG, Control Group

Table 1. General characteristics of the subjects.

\begin{tabular}{|c|c|c|c|c|c|c|c|}
\hline & \multicolumn{3}{|c|}{ EG $(n=10)$} & \multicolumn{3}{|c|}{$C G(n=10)$} & \multirow[b]{2}{*}{$P^{\mathrm{a}}$} \\
\hline & pre & post & $p$ & pre & post & $p$ & \\
\hline SEF & $11.70 \pm 6.02$ & $16.60 \pm 5.54$ & $0.01^{*}$ & $14.00 \pm 4.19$ & $15.80 \pm 4.00$ & $0.00^{*}$ & $0.02^{*}$ \\
\hline Wrist & $3.60 \pm 3.95$ & $4.20 \pm 3.55$ & 0.18 & $4.90 \pm 4.18$ & $5.30 \pm 4.08$ & 0.41 & 0.74 \\
\hline Hand & $6.00 \pm 4.27$ & $8.40 \pm 2.80$ & $0.01^{*}$ & $6.60 \pm 5.34$ & $7.60 \pm 5.23$ & $0.02^{*}$ & $0.04^{*}$ \\
\hline Coordination & $2.40 \pm 0.70$ & $3.40 \pm 0.70$ & $0.02^{*}$ & $3.70 \pm 2.00$ & $3.40 \pm 1.90$ & 0.52 & $0.03^{*}$ \\
\hline
\end{tabular}

$\overline{\mathrm{M} \pm \mathrm{SD} ;}{ }^{*}, P<0.05 ; P^{a}$, post-pre test in between the EG and $\mathrm{CG}$; SEF, Shoulder/Elbow/Forearm; EG, Experimental Group; CG, Control Group 
recover the upper extremity functions, and therapists and patients with stroke need to make a lot of efforts to achieve the upper limb functions. ${ }^{2)}$ The motor output depends on how much sensory input comes in to the brain and that should be precise and clear. Likewise, the sensory receptions rely on how accurately each segment of the body moves and should be individual and segmental movement. When it comes to balance, the sensory input and motor output are necessary and essential, and these should be coordinated all the times to the diverse environment. For instance, when they grasp an object on a desk, first they need to get the information on where it is and what shape it is through the visual contact on it. Then, the visual information comes into the occipital lobe in where the vision area is and integrate with the previous memory coming from sensory associated areas resulting in making them be ready to grasp. On the top of that, during grasping the object, somatosensory and proprioception information from muscles which are involved in this movement are conveyed to the motor system helping the movement more efficient and precise. More importantly, ongoing sensory and motor feedback and feedforward have to be proceeded for proper and accurate movement and balance control. ${ }^{5)}$ Thus, the primary motor and somatosensory areas in the brain make their shapes in accordance with peripheral somatosensory information and actual movement. ${ }^{12)} \mathrm{A}$ way to reinforce motor function depends on activating the peripheral somatosensory system. The previous study proved that combining repetitive peripheral sensory stimulation of the affected upper limb with exercises enhanced motor output in patients after stroke. ${ }^{13)}$ In this study, the protocol and sequence of the intervention were based on the motor control which emphasizes sensory input for motor outcome. What that means is that the first step in the intervention was give the subjects tactile and proprioceptive information in the intrinsic muscles by lengthening the individual muscles and facilitating muscle spindles and golgi tendon organ in it. Thus, the ample and diverse sensory information were transmitted to the primary somatosensory area and integrated with the previous memory about the stim- ulation and information coming from the hand. As a result, there are significant differences in the hand, forearm, elbow and shoulder functions after the intervention. Therefore, the subjects in this study got benefits from the first step provided in the intervention which is activation of the intrinsic muscles in the affected side hand. The previous study provided multiple interventions from deliberate anticipation to attentive exploration of stimuli to quantitative feedback to gradual discrimination activities. As a result, all subjects got improved of trained abilities and the function of the affected hand turned out to be comparable to those of the less affected hand. Moreover, these therapeutic effects were preserved for up to 5 months. ${ }^{14)}$ In the previous study, systematic rehabilitation of the sensory function was applied to the subjects and practicing tasks was followed by 3 times a week for 6 weeks. The treated group ended up large and big gains in sensory tests (stereognosis, tactile location, 2-point discrimination, elbow proprioception) which is very meaningful because the subjects in the study performed activities after getting the treatment with sensory stimuli. ${ }^{6}$ In addition, the previous study which provided not only training of somatosensation but also exercises focused on sensory-related dysfunction of motor control. The results of the study displayed by using a wide-range rehabilitative protocol, a significant degree of generalization of acquired capacities can be achieved. ${ }^{1)}$ In this study, the protocol of the intervention focused on the somatosensory stimuli through the affected hand by giving the tactile information and proprioception from the therapist hands in order to get the subjects more awareness on body schema of the hand in the brain. Moreover, as getting the intrinsic muscles in the affected hand proper length, the hand can have more opportunities to get wider range of motion in the hand resulting in getting stronger muscle strength. In addition, the stability on the affected shoulder was targeted in the process of the intervention. This is because the shoulder joint plays a very important role on orientating the direction where the hand is supposed to go to. When people try to grasp an object, the hand which is supposed to grasp open properly 
according to the shape and size of the object. And then, the shoulder moves over towards where the object is. With proper stability of the shoulder, we can reach the hand all the way out to reach the object. That is way the scapular setting was considered in the treatment and applied as a second step of the intervention. And, through manipulation in the air, the subjects had more chances to get more accurate proprioception on the shoulder, elbow and wrist joints. At the same time, the subjects were able to practice coordination of the reaching movement and manipulating an object in the affected hand reinforced the shoulder stability and sensory input in the affected hand. Like this, in this study, the intervention started with sensory input and ended in motor output. Most importantly ongoing repetition of sensory input in combination with active movement brought improved upper limb functions.

\section{References}

1. Smania N, Montagnana B, Faccioli S, et al. Rehabilitation of somatic sensation and related deficit of motor control in patients with pure sensory stroke. Arch Phys Med Rehabil. 2003;84(11):1692-702.

2. Ghaziani E CC, Henkel et al. Electrical somatosensory stimulation followed by motor training of the paretic upper limb in acute stroke: study protocol for a randomized controlled trial. Trials. 2017;18(1):84.

3. Tu-Chan AP, Natrai N, Godlove J, et al. Effects of somatosensory electrical stimulation on motor function and cortical oscillations. J Neuroeng Rehabil. 2017;14(1):113.

4. Lämås $\mathrm{K}$, Häger $\mathrm{C}$, Lindgren $\mathrm{L}$, et al. Does touch massage facilitate recovery after stroke? A study protocol of a randomized controlled trial. BMC Complement Altern Med. 2016;4(16):50.
5. Borich MR, Brodie SM, Gray WA, et al. Understanding the role of the primary somatosensory cortex: Opportunities for rehabilitation. Neuropsychologia. 2015;79(Pt B): 246-55.

6. Yekutiel M, Guttman E. A controlled trial of the retraining of the sensory function of the hand in stroke patients. J Neurol Neurosurg Psychiatry. 1993;56(3):241-4.

7. Raine S ML, Lynch-Ellerington M. The Bobath Concept: Theory and clinical practice in neurological rehabilitation, 1st edition. 2013.

8. Mottram SL. Dynamic stability of the scapula. Man Ther. 1997;2(3):123-31.

9. Kandel ER SJ, Jessel TM. Principle of Neural Science, 4th edn. New York: McGraw-Hill; 2000.

10. Jeannerod M. Visuomotor channels: Their integration in goal directed prehension. Human Movement Sci. 1999;18:201-18

11. Sanford J, Moreland J, Swanson LR, et al. Reliability of the Fugl-Meyer assessment for testing motor performance in patients following stroke. Phys Ther. 1993;73(7):447-54.

12. Hamdy S, Rothwell JC, Aziz Q, et al. Long-term reorganization of human motor cortex driven by short-term sensory stimulation. Nat neurosci. 1998;1(1):64-8.

13. Knutson JS, Harley MY, Hisel TZ, et al. Contralaterally controlled functional electrical stimulation for stroke rehabilitation. Conf Proc IEEE Eng Med Biol Soc. 2012;314-7.

14. Carey LM, Matyas TA, Oke LE. Sensory loss in stroke patients: effective training of tactile and proprioceptive discrimination. Arch Phys Med Rehabil. 1993;74(6): 602-11. 
